\title{
Mediastinal lymphadenectomy during robotic pulmonary resection
}

\section{Farid Gharagozloo}

Center for Advanced Thoracic Surgery, Global Robotics Institute, Advent Health Celebration, University of Central Florida, Celebration, Florida, USA Correspondence to: Farid Gharagozloo, MD, FACS. Center for Advanced Thoracic Surgery, 400 celebration Place, Celebration, Celebration, Florida 34786, USA. Email: Gharagozloof@aol.com.

\begin{abstract}
Concerns for oncologic outcomes have played a significant role in the resistance to a more widespread adoption of video-assisted thoracoscopic surgical (VATS) approach to anatomical lung resections. VATS lobectomy has consistently accounted for $20-30 \%$ of lobectomy procedures. The advent of robotics has been a significant factor in a more widespread adoption of minimally invasive techniques for lung resection by an increasing number of surgeons. The surgical robot facilitates precise instrument maneuverability in a confined space and provides high resolution three-dimensional visualization of the anatomy. During anatomic lung resection, the robotic approach allows for replication of the time-honored technique through a thoracotomy. Furthermore, robotics allows for wide dissection of the mediastinum in performing a complete mediastinal nodal dissection as opposed to sampling. The wide mediastinal and hilar dissection in turn facilities accurate anatomic resection of the involved lobe. Finally, an important factor in the more widespread adoption of the robotic technique appears to be the greater efficacy of mediastinal and hilar nodal dissection and pathologic upstaging in patients with operable non-small cell lung cancer. The overall pathologic upstaging after mediastinal nodal dissection with robotic-assisted lobectomy has been reported to be $24 \%$ with $18 \% \mathrm{~N} 1$ upstaging and $6 \% \mathrm{~N} 2$ upstaging. This is similar to upstaging results with lobectomy by thoracotomy (14.3-24.6\%) and higher than VATS (10.6-11.9). This communication outlines the technique and results of mediastinal and hilar lymphadenectomy during robotic-assisted lung resection.
\end{abstract}

Keywords: Robotic; lobectomy; mediastinal nodal dissection; lymphadenectomy; lung cancer

Received: 30 June 2021; Accepted: 07 September 2021; Published: 30 March 2022.

doi: $10.21037 /$ asj-21-56

View this article at: https://dx.doi.org/10.21037/asj-21-56

\section{Introduction}

Anatomic pulmonary resection with concomitant mediastinal and hilar nodal dissection is the standard of care for the surgical treatment of patients with early-stage non-small cell lung cancer (NSCLC) (1). Video-assisted thoracoscopic lobectomy (VATS) has been shown to be associated with reduced morbidity when compared to lobectomy by thoracotomy (2). However, VATS is hampered by limited instrument maneuverability, two-dimensional visualization, and technical challenges, and a steep learning curve in relation to the mediastinal hilar dissection (3). Roboticassisted lobectomy overcomes these limitations by providing precise instrument maneuverability in a confined space and three-dimensional visualization. An additional advantage of the robotic approach may be more precise hilar dissection and more complete mediastinal lymph node dissection which can potentially improve staging and optimize postoperative oncologic treatment strategy (4-6).

This paper outlines the approach to mediastinal and hilar nodal dissection in patients undergoing roboticassisted atomic lung resection for operable lung cancer. All procedures performed in this study were in accordance with the ethical standards of the institutional research committee and with the Helsinki Declaration (as revised in 2013). Written informed consent was obtained from the patient for publication of article and accompanying images. A copy of the written consent is available for review by the editorial office of this journal.

\section{Surgical technique}

Double-lumen tube is used for lung isolation and the 


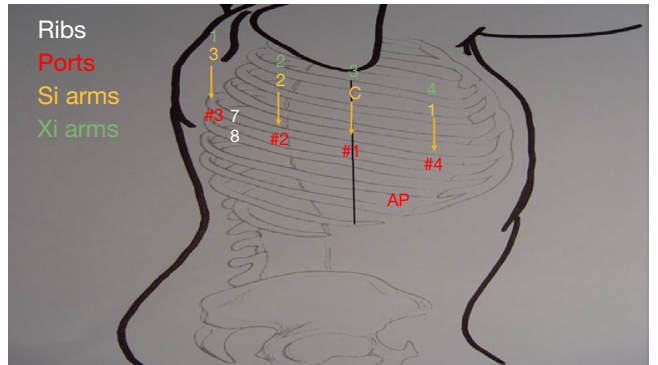

Figure 1 Port placement right chest. AP, accessory port.

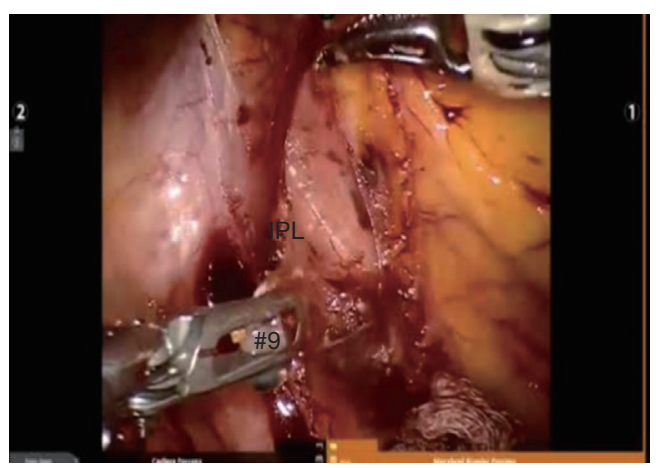

Figure 2 Intraoperative photograph of right sided lymphadenectomy. Inferior pulmonary ligament (IPL) has been divided and the station \#9 node is being removed.

patient is positioned in a full lateral decubitus position. The right arm is placed over pillows and positioned high enough such that access to the fourth intercostal space (ICS) in the anterior axillary line is readily attained. The table is flexed in order to move the hip down and to open the ICSs. The lung is deflated and placed on suction. The position of the double lumen tube is rechecked after the patient is prepped and draped.

\section{Mediastinal and hilar nodal dissection during right sided robotic lobectomy}

A line is drawn from the tip of the scapula to the costal arch. This delineates the highest point in the chest and the midscapular line (posterior axillary line) (Figure 1). Pleural entry is with a Hassan needle. Saline is infused and care is taken to look for easy egress of the saline from the needle. Port \#1 is the camera port and is placed in the $7^{\text {th }}$ ICS. Warm, humidified $\mathrm{CO}_{2}$ is insufflated through this port at a flow of $6 \mathrm{~L} / \mathrm{min}$ to a pressure of $6-8 \mathrm{mmHg}$ in order to push the lung and diaphragm away. The other ports are placed under direct vision. Port \#2 $(8 \mathrm{~mm})$ is placed in the seventh ICS in the poster scapular line. This port is $9 \mathrm{~cm}$ posterior to port \#1 and accommodates da Vinci Si and Xi arm \#2. Prior to the placement of port \#3, a 21-gauge needle is inserted into the seventh ICS at costovertebral junction from the patient's back and injects a $10-\mathrm{mL}$ subpleural bubble of $0.25 \%$ bupivacaine with epinephrine near the intercostal nerve. Next, port \#3 is placed $10 \mathrm{~cm}$ posterior to port \#2 in the seventh ICS just medial to the spine. This port accommodates da Vinci Si arm \#3 or Xi arm \#1. Port \#4 is placed $9 \mathrm{~cm}$ anterior to port \#1 in the seventh ICS at the anterior scapular line. This port accommodates da Vinci Si arm \# 1 or $\mathrm{Xi}$ arm \#4. The assistant port \#5 uses a 10-12 Versaport trocar and is placed in the ninth ICS and is triangulated between port \#1 and \#4. It should be two or three rib spaces lower than and as distant to the da Vinci ports as possible to maximize assistant workspace.

Review of port placement with Si robot: camera is inserted through port \#1. Robotic arm \#3 is placed through port \#3. Robotic arm \#2 is placed through port \#2. Robotic arm \#1 is placed through port \#4.-

Review of port placement with Xi robot: robotic arm \#1 is placed through port \#3. Robotic arm \#2 is placed through port \#2. Robotic arm \#4 is placed through port \#4. The assistant port is triangulated behind the camera arm and robotic arm \#4 in a similar fashion. The camera is carried by arm \#3 and placed through port \#1.-

Camera is $0^{\circ}$ and/or $30^{\circ}$ down viewing endoscope. Instruments consist of a 5 -mm thoracic grasper or Cadiere forceps in the left hand, and Curved Bipolar Dissector in the right hand.

Mediastinal and hilar nodal and hilar dissection is the same for all lobectomies. The inferior pulmonary ligament is divided and the nodes from station \#9 and \#8 are removed (Figure 2). Next the pleura anterior to the vagus nerve is opened, and the anterior branch of the nerve which traverses the subcarinal space is divided. The right mainstem bronchus is identified and station \#7 nodes are removed while staying posterior to the edge of the cartilage of the right mainstem bronchus. At the end of the dissection the right and left mainstem bronchi should be visible and the posterior aspect of the pericardium should be cleaned and clearly visible (Figure 3). Next, the most posterior arm is used to retract the upper lobe inferiorly in order to facilitate the dissection of nodal stations $2 \mathrm{R}$ and $4 \mathrm{R}$. These nodes are removed by opening the pleura just posterior to the superior vena cava and clearing the space between the superior vena cava (SVC) anteriorly, the trachea 


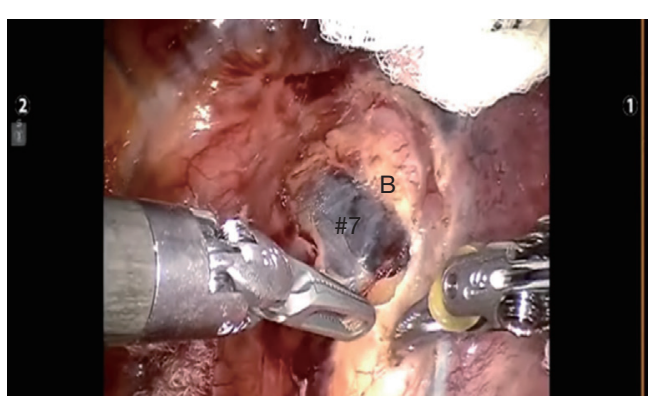

Figure 3 Intraoperative photograph of right sided lymphadenectomy. Station \#7 nodes are removed from underside of the right mainstem bronchus (B) in completing the subcarinal lymphadenectomy.

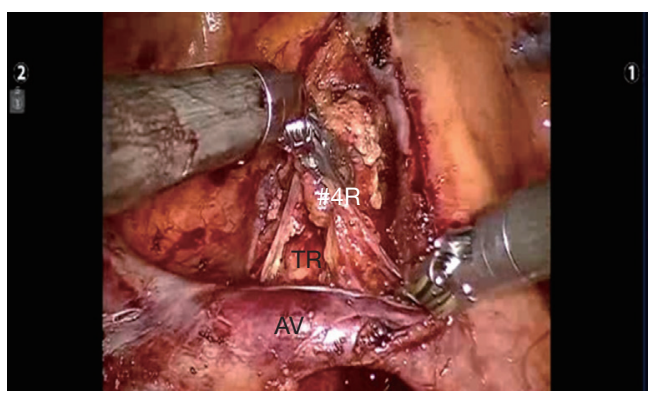

Figure 4 Intraoperative photograph of right sided lymphadenectomy. Station \#4 nodes are approached by clearing the space between the superior vena cava, the azygous vein (AV) and the trachea (TR).

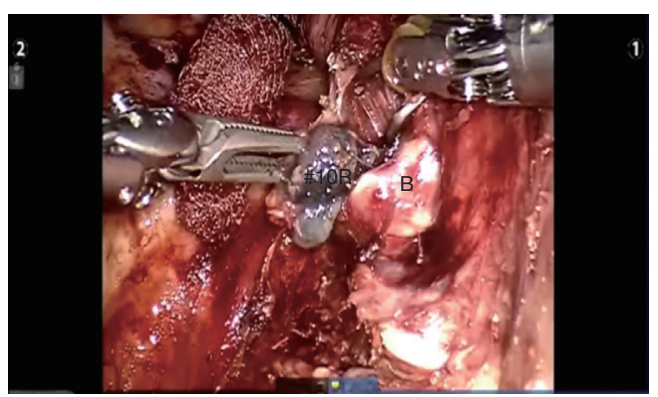

Figure 5 Intraoperative photograph of right sided lymphadenectomy. Station \#10 nodes are removed from the space above the right upper lobe bronchus (B).

posteriorly, and the azygos vein inferiorly (Figure 4). Station \#10R lymph node is removed after identifying the right mainstem bronchus and following it to the nodal station. This node can sit between the truncus branch and the superior pulmonary vein, adjacent to the posterior aspect of the bronchus or even anterior to the superior pulmonary vein

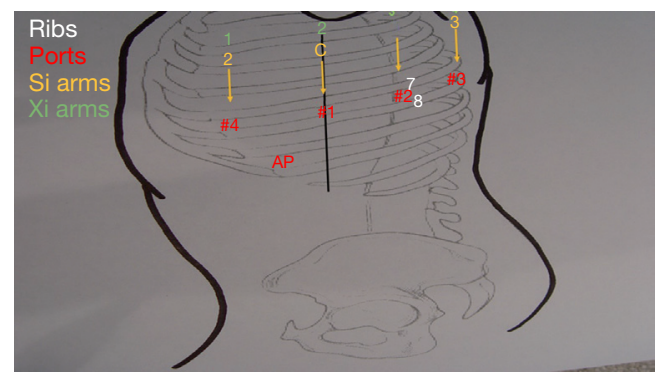

Figure 6 Port placement left chest. AP, accessory port.

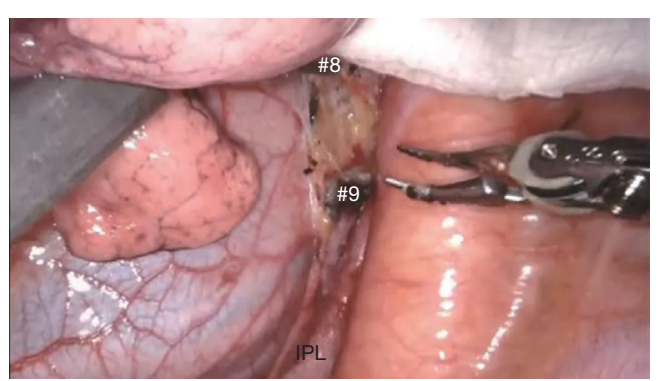

Figure 7 Intraoperative photograph of left sided lymphadenectomy. Inferior pulmonary ligament (IPL) has been divided and the station \#8 and \#9 nodes is being removed.

(Figure 5). Completion of the lymph node dissection opens the mediastinal space and facilitates the dissection of the artery and the bronchus for the completion of the lobectomy.

\section{Mediastinal and bilar nodal dissection during left sided lobectomy}

Port placement for $\mathrm{Si}$ and $\mathrm{Xi}$ robot is shown in Figure 6. The placement of the robot and instruments are the same as the right sided approach. For all lobectomies, the dissection begins with mediastinal nodes. The nodal dissection is the same for any lobectomy. The inferior pulmonary ligament is divided and station \#9 and \#8 nodes are removed (Figure 7). The lung is retracted medially and anteriorly in order to remove lymph nodes from station \#7. The pleura anterior to the vagus nerve is opened. The left mainstem bronchus is identified and the dissection is confined to the area inferior to the edge of the bronchial cartilage. The station \#7 nodal bundle is accessed between the inferior pulmonary vein and the left mainstem bronchus. The nodal bundle is traced to the carina and is then removed (Figure 8). Next the lung is retracted inferiorly and the pleura overlying station \#4 nodal bundle is opened. Station \#4L nodes are removed 


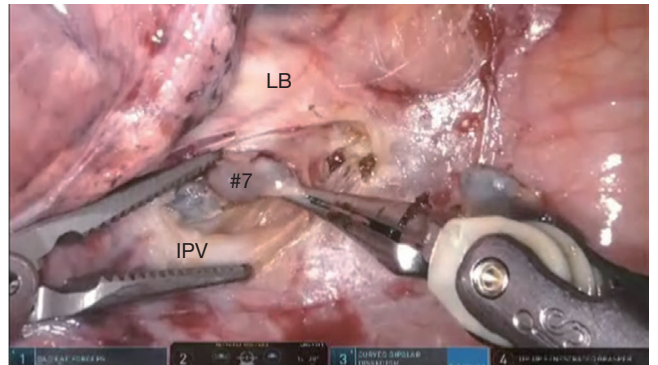

Figure 8 Intraoperative photograph of left sided lymphadenectomy. Station \#7 nodes are removed from underside of the left mainstem bronchus (LB) and just superior to the inferior pulmonary vein (IPV).

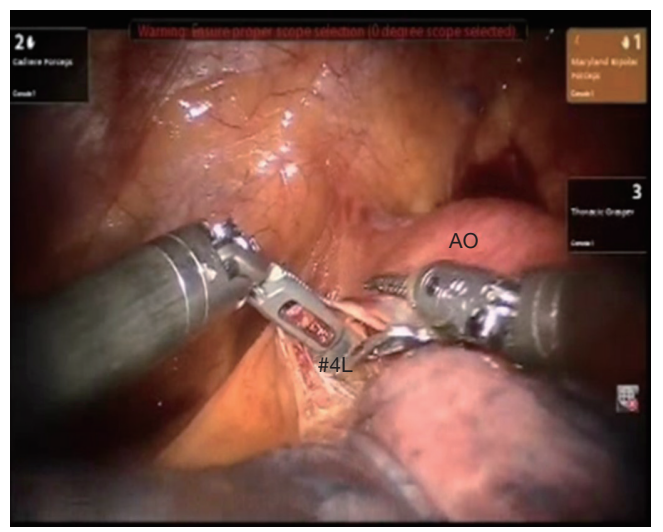

Figure 9 Intraoperative photograph of left sided lymphadenectomy. Station \#4 nodes are removed from the space between the trachea and the aortic arch (AO).

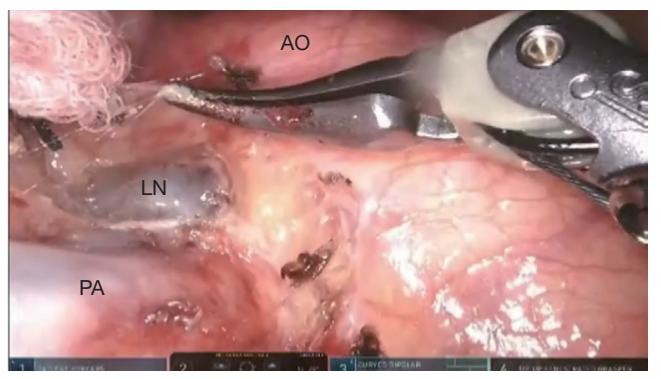

Figure 10 Intraoperative photograph of right sided lymphadenectomy. The space between the pulmonary artery (PA) and the aorta $(\mathrm{AO})$ is cleared in order to visualize the nodal bundle which encases the apicoposterior trunk of the pulmonary artery. LN, lymph node.
(Figure 9). During this dissection, are should be exercised to distinguish what may actually be station \#10L or even station 5 lymph nodes in this area. The left main pulmonary artery is identified above the left main bronchus. The space between the pulmonary artery and the bronchus is opened, and station \#10L nodal bundle is identified overlying the superior border of the bronchus. The space between the pulmonary artery and the aorta is cleared in order to visualize the nodal bundle which can be located adjacent to the apico-posterior trunk of the artery or anterior to the superior vein (Figure 10) Care is taken to identify and preserve the vagus and the recurrent laryngeal branch. After exposing the apico-posterior trunk, the nodal bundle (station \#10) is swept in an infero-medial direction thereby exposing the underside of the truncus branch and its takeoff from the main pulmonary artery. There may be additional station \#10 lymph nodes adjacent to the apico-posterior trunk of the artery, but these may actually be station \#12 lymph nodes if located between the underside of the truncus branch and the main pulmonary artery. The methodical nodal dissection facilitates exposure of the bronchovascular structures in preparation for the lung resection.

\section{Comments}

An accurate and thorough mediastinal and hilar nodal dissection is crucial for determining the pathologic stage and may be a significant factor in determining the postoperative oncologic treatment strategies in patients with operable NSCLC. In addition, during robotic-assisted lobectomy, thorough mediastinal and hilar nodal dissection is an essential step in achieving safe and complete anatomic lung resection by exposing critical anatomic structures and spaces during the conduct of operation.

The median number of lymph nodes harvested during lobectomy by thoracotomy is 15 (7). Similar results have been reported for VATS Lobectomy. With robotic lobectomy the median number of nodes harvest has been reported to range from 14 to 18 . However, overall pathologic upstaging after mediastinal nodal dissection with robotic-assisted lobectomy has been reported to be $24 \%$ with $18 \%$ N1 upstaging and $6 \% \mathrm{~N} 2$ upstaging $(8,9)$. This is similar to upstaging results with lobectomy by thoracotomy (14.3-24.6\%) and higher 
than VATS (10.6-11.9\%) (8). In a retrospective assessment of 249 patients undergoing robotic-assisted lobectomy for lung cancer $63.9 \%$ of patients were clinical stage 1 prior to lung resection. This dropped to $53.8 \%$ after pathologic assessment. Stage-specific survival was positively impacted by upstaging with the robotic approach (10).

Undoubtedly the use of robotic technology adds more ports, and results in greater cost. The "greater cost" associated with robotic technology is due potentially to capital purchase of the robotic system and to the disposable robotic arm coverings and reposable robotic instruments. However, the overall cost may be decreased due to shorter hospital stays compared to thoracotomy and potentially fewer conversions to thoracotomy compared to VATS lobectomy. In addition, in the long term, these shortcomings may be obviated by the potentially more efficacious oncologic approach to lung resection and mediastinal and hilar nodal dissection afforded by the robot. In turn greater oncologic efficacy may be a significant factor in adopting minimally invasive approaches to the treatment of patients with operable lung cancer.

\section{Acknowledgments}

Funding: None.

\section{Footnote}

Provenance and Peer Review: This article was commissioned by the Guest Editor (Hitoshi Igai) for the series "Lymphadenectomy in Thoracoscopic Surgery" published in AME Surgical fournal. The article has undergone external peer review.

Conflicts of Interest: The author has completed the ICMJE uniform disclosure form (available at https://asj.amegroups. com/article/view/10.21037/asj-21-56/coif). The series "Lymphadenectomy in Thoracoscopic Surgery" was commissioned by the editorial office without any funding or sponsorship. The author has no other conflicts of interest to declare.

Ethical Statement: The author is accountable for all aspects of the work in ensuring that questions related to the accuracy or integrity of any part of the work are appropriately investigated and resolved. All procedures performed in this study were in accordance with the ethical standards of the institutional research committee and with the Helsinki Declaration (as revised in 2013). Written informed consent was obtained from the patient for publication of article and accompanying images. A copy of the written consent is available for review by the editorial office of this journal.

Open Access Statement: This is an Open Access article distributed in accordance with the Creative Commons Attribution-NonCommercial-NoDerivs 4.0 International License (CC BY-NC-ND 4.0), which permits the noncommercial replication and distribution of the article with the strict proviso that no changes or edits are made and the original work is properly cited (including links to both the formal publication through the relevant DOI and the license). See: https://creativecommons.org/licenses/by-nc-nd/4.0/.

\section{References}

1. Ginsberg RJ, Rubinstein LV. Randomized trial of lobectomy versus limited resection for T1 N0 non-small cell lung cancer. Lung Cancer Study Group. Ann Thorac Surg 1995;60:615-22; discussion 622-3.

2. Nakata $M$, Saeki H, Yokoyama N, et al. Pulmonary function after lobectomy: video-assisted thoracic surgery versus thoracotomy. Ann Thorac Surg 2000;70:938-41.

3. Velez-Cubian FO, Ng EP, Fontaine JP, et al. RoboticAssisted Videothoracoscopic Surgery of the Lung. Cancer Control 2015;22:314-25.

4. Velez-Cubian FO, Rodriguez KL, Thau MR, et al. Efficacy of lymph node dissection during robotic-assisted lobectomy for non-small cell lung cancer: retrospective review of 159 consecutive cases. J Thorac Dis 2016;8:2454-63.

5. Detterbeck FC, Boffa DJ, Kim AW, et al. The Eighth Edition Lung Cancer Stage Classification. Chest 2017;151:193-203.

6. Goldstraw P, Chansky K, Crowley J, et al. The IASLC Lung Cancer Staging Project: Proposals for Revision of the TNM Stage Groupings in the Forthcoming (Eighth) Edition of the TNM Classification for Lung Cancer. J Thorac Oncol 2016;11:39-51.

7. Darling GE, Allen MS, Decker PA, et al. Randomized trial of mediastinal lymph node sampling versus complete lymphadenectomy during pulmonary resection in the patient with $\mathrm{N} 0$ or $\mathrm{N} 1$ (less than hilar) non-small cell carcinoma: results of the American College of Surgery Oncology Group Z0030 Trial. J Thorac Cardiovasc Surg 2011;141:662-70.

8. Park BJ, Melfi F, Mussi A, et al. Robotic lobectomy for non-small cell lung cancer (NSCLC): long-term oncologic 
results. J Thorac Cardiovasc Surg 2012;143:383-9.

9. Casiraghi M, Galetta D, Spaggiari L. Robotic assisted lobectomy and lymphadenectomy "different approaches". Shanghai Chest 2018;2:17.

doi: $10.21037 /$ asj-21-56

Cite this article as: Gharagozloo F. Mediastinal lymphadenectomy during robotic pulmonary resection. AME Surg J 2022;2:9.
10. Toosi K, Velez-Cubian FO, Glover J, et al. Upstaging and survival after robotic-assisted thoracoscopic lobectomy for non-small cell lung cancer. Surgery 2016;160:1211-8. 